\title{
openheart The impact of an integrated heart failure service in a medium-sized district general hospital
}

\author{
Alasdair Hawley (D) , ${ }^{1,2}$ Jingzhou He, ${ }^{3}$ Alice Crabtree,${ }^{4}$ Stelios lacovides, ${ }^{1}$ \\ Phil Keeling ${ }^{1}$
}

To cite: Hawley A, He J, Crabtree A, et al. The impact of an integrated heart failure service in a medium-sized district general hospital. Open Heart 2020;7:e001218. doi:10.1136/ openhrt-2019-001218

Received 6 January 2020 Revised 26 February 2020 Accepted 27 March 2020

\section{Check for updates}

(c) Author(s) (or their employer(s)) 2020. Re-use permitted under CC BY-NC. No commercial re-use. See rights and permissions. Published by BMJ.

${ }^{1}$ Cardiology, Torbay and South Devon NHS Foundation Trust, Torquay, UK

${ }^{2}$ Medicine, Royal Devon and Exeter NHS Foundation Trust, Exeter, UK

${ }^{3}$ Cardiology, Royal Devon and Exeter NHS Foundation Trust, Exeter, UK

${ }^{4}$ Medicine, Torbay and South Devon NHS Foundation Trust, Torquay, UK

\section{Correspondence to}

Dr Alasdair Hawley; alasdair. hawley@nhs.net

\section{ABSTRACT}

Objectives Assessing the impact of a new integrated heart failure service (IHFS) in a medium-sized district general hospital (DGH) on heart failure (HF) mortality, readmission rates, and provision of $\mathrm{HF}$ care.

Methods A retrospective, observational study encompassing all patients admitted with a diagnosis of $\mathrm{HF}$ over two 12-month periods before (2012/2013), and after (2015/2016) IHFS establishment.

Results Total admissions for HF increased by $40 \%$ (385 vs 540 ), with a greater number admitted to the cardiology ward (231 vs 121). After IHFS implementation, patients were more likely to see a cardiologist (35.1\% vs $43.7 \%$, $p=0.009)$, undergo echocardiography $(70.1 \%$ vs $81.5 \%$, $\mathrm{p}<0.001$ ), be initiated on all three disease modifying HF medications (angiotensin-converting enzyme inhibitors (ACEi), angiotensin II receptor blockers (ARB) and mineralocorticoid receptor antagonists (MRA)) in the heart failure with reduced ejection fraction (HFrEF) group (42\% vs $99 \%, p<0.001)$ and receive specialist HF input $(81.6 \%$ vs $85.4 \%, p=0.2)$. Both 30 -day post-discharge mortality and $\mathrm{HF}$ related readmissions were significantly lower in patients with heart failure with preserved ejection fraction (HFpEF) $(8.9 \%$ vs $3.1 \%, p=0.032,58 \%$ reduction, $p=0.043$ respectively) with no-significant reductions in all other HF groups. In-patient mortality was similar. Length of stay in Cardiology wards increased from 8.4 to 12.7 days $(p<0.001)$.

Conclusion Establishment of an IHFS within a DGH with limited resources and only a modest service re-design has resulted in significantly improved provision of specialist in-patient care, use of HFrEF medications, early heart failure nurse follow-up, and is associated with a reduction in early mortality, particularly in the HFpEF cohort, and HF related readmissions.

\section{INTRODUCTION}

Heart failure (HF) is a complex clinical syndrome characterised by symptoms and signs caused by a structural and/or functional cardiac abnormality, leading to a reduction in cardiac output and/or elevated intracardiac pressures. ${ }^{12}$ In the UK, an estimated 900000 people live with $\mathrm{HF}$, and it remains one of the leading causes of recurrent hospital admissions, particularly in the elderly. ${ }^{3}$ In

\section{Key questions}

What is already known about this subject?

- Heart failure (HF) prevalence is increasing and it is a major economic burden to the national health service (NHS). European society of cardiology (ESC) ${ }^{9}$ and the national institute for health and care excellence (NICE) guidelines recommend a multidisciplinary HF specialist team approach to reduce mortality and hospitalisation. However, there is very little data demonstrating the effectiveness of this approach in a real world setting within a district general hospital (DGH).

What does this study add?

- This study shows that the introduction of an integrated heart failure service (IHFS) into a medium-sized DGH can lead to better patient care, with statistically significant reductions in 30-day mortality and HFrelated readmissions in patients with $\mathrm{HFpEF}$.

How might this impact on clinical practice?

- Management of HF by an IHFS improves clinical care and patient outcome. An IHFS can be established with modest resources and limited service redesign. We believe this model of care should become routine throughout the NHS.

2014-2015, the 'national heart failure audit' reported nearly 57000 admissions in England and Wales, with an average length of stay of 11 days at a cost of approximately $£ 3000$ per admission. In-hospital mortality was $9.6 \%$, 30 -day mortality $20 \%$ and mortality at 1 year was $30 \%{ }^{4}$

Torbay and South Devon covers an area of 350 square miles and includes both rural communities and urban centres. It is a medium-sized district general hospital (DGH) within an integrated organisation and provides acute healthcare services to a resident population of approximately 300 000 , increasing by up to 100000 during the holiday season. ${ }^{5}$ Torbay's population is elderly with $26.5 \%$ over 65 years of age (England national average $18.1 \%$ ) and this proportion 
is expected to increase to $32 \%$ by 2030 . In addition, it is the most deprived local authority area in the South West region. People in deprived communities tend to experience multiple long-term conditions and generally have poorer health outcomes. ${ }^{6}$

With the publication of NICE guidelines on the management of $\mathrm{HF}$ in $2010,{ }^{7}$ we began to develop an integrated heart failure service (IHFS) for patients within Torbay hospital and across the wider healthcare community. The service was introduced in May 2013 with the aims of improving the following:

- Use of HFrEF medications known to improve survival.

- Education for patients and their families.

- Provision of home-based care.

- Continuity and coordination of care.

- Joined-up working between specialties (palliative care, elderly care, renal, intermediate care).

- Clinical outcomes (reduce mortality and readmissions).

The IHFS takes a multidisciplinary approach and comprises two cardiologists with an interest in HF, five specialist heart failure nurses (HFNs) (two were already employed within primary care and three (2.4 whole-timeequivalent (WTE)) were new appointments) together with administrative and audit support. HFN training involved an 18-month rotation between the hospital and community prior to establishment of a single hospitalbased, and four community-based HFNs (total 4.4 WTE).

The IHFS actively identifies patients admitted with suspected HF using B-type natriuretic peptide (BNP) and automated email alerts for patients with known HF. The HFNs review these patients and discuss them with the 'cardiologist of the week'. Together, they identify patients suitable for transfer to the cardiology ward. Furthermore, all in-patients are discussed formally at the weekly HF multidisciplinary team (MDT). This provides all patients with specialist consultant input, either by face-to-face review on the HF ward, or in discussion with the HFNs.

Following discharge (pre-IHFS and post-IHFS), patients are scheduled for urgent outpatient consultant clinic review, which usually occurs within 6 weeks (due to high demand). However, the new IHFS aims to also provide specialist HFN review in the community for all HF patients within 2 weeks of discharge.

There is particular focus on improving coding, confirming subtype, providing patient education and self-management, providing early HFN review post discharge and follow-up, and ensuring a seamless transition between hospital and community services.

\section{METHOD}

Torbay hospital has participated in the national HF audit (NICOR) since its inception in 2007 and over this period, has established an effective audit process for patients coded as having HF on admission. The diagnosis is confirmed when a patient presents with appropriate signs and symptoms, with echocardiographic abnormality and elevation in N-terminal B-type pro B-type natriuretic peptide (NT pro-BNP). In those patients who have not had an in-patient or recent ( $<12$ months) echocardiogram, clinical assessment with the aid of Chest X-ray (CXR) and 12-lead ECG is made.

All admissions with a coded diagnosis of $\mathrm{HF}$ were reviewed for each 12-month period (01/04/2012$31 / 03 / 2013, \quad \mathrm{n}=420$ and $01 / 04 / 2015-31 / 03 / 2016$, $\mathrm{n}=576$ ) (see table 1 ). Some admissions were readmissions $<30$ days (35 and 36 , respectively) and were excluded from the analysis (as these would be represented in the final readmission rates). Data from the remaining patients were attained by review of medical notes, echocardiograms, blood results and information from the integrated health care system (IHCS). Of total admissions, 350 patients in 2012/2013 and 505 patients in 2015/2016 had undergone echocardiography allowing HF subtype to be confirmed (HFrEF-ejection fraction $\leq 40 \%$ without prior significant valve disease, HFpEFejection fraction $\geq 40 \%$ with evidence of diastolic dysfunction, and valvular heart failure (VHF)-presence of at least moderate-severe aortic or mitral valve disease). Analysis of in-patient deaths and readmissions within 30 days of discharge was undertaken to confirm the cause of death and the clinical reason for readmission. In some cases this required additional discussion with the General Practitioner (GP).

\section{Statistical analysis}

Statistical analysis of mortality and readmission rates was performed using the $\chi^{2}$ test. Length-of-stay analysis was performed using a non-related t-test. Due to the singlecentre nature of this study and relatively small patient numbers, statistical power is decreased.

\section{Patient and public involvement}

As a retrospective, observational study of a service re-design, patients and the public were not directly involved in the design of this study.

\section{RESULTS}

Table 1 shows the total of suspected and confirmed HF admissions for the 2012/2013 and 2015/2016 cohorts. Over this period, admissions increased $40 \%$ from 385 to 540 patients. Patient morbidity data is shown in table 2. Patients in the two cohorts were of similar age but in the 2015/2016 cohort were more symptomatic on admission (New York Heart Association (NYHA) 3 or 4, 89.4\% vs $74.5 \%)$, more co-morbid, and had worse renal function (mean serum creatinine $129 \mathrm{vs} 123 \mathrm{mmol} / \mathrm{L}$ ). There were also more likely to be admitted to a cardiology ward $(31.4 \%$ vs $42.8 \%, \mathrm{p}<0.001)$, be seen by a cardiologist ( $35.1 \%$ vs $43.7 \%, \mathrm{p}=0.009)$, receive specialist HF in-patient care $(81.6 \%$ vs $85.4 \%, \mathrm{p}=0.12)$, undergo echocardiography $(70.1 \%$ vs $81.5 \%, \mathrm{p}<0.001)$, and receive an early post-discharge home visit from an HFN (28\% vs $57 \%$, $\mathrm{p}<0.001$ ) (table 3). In the HFrEF group, the use of all three disease modifying HF medications (ACE-/ARB, 


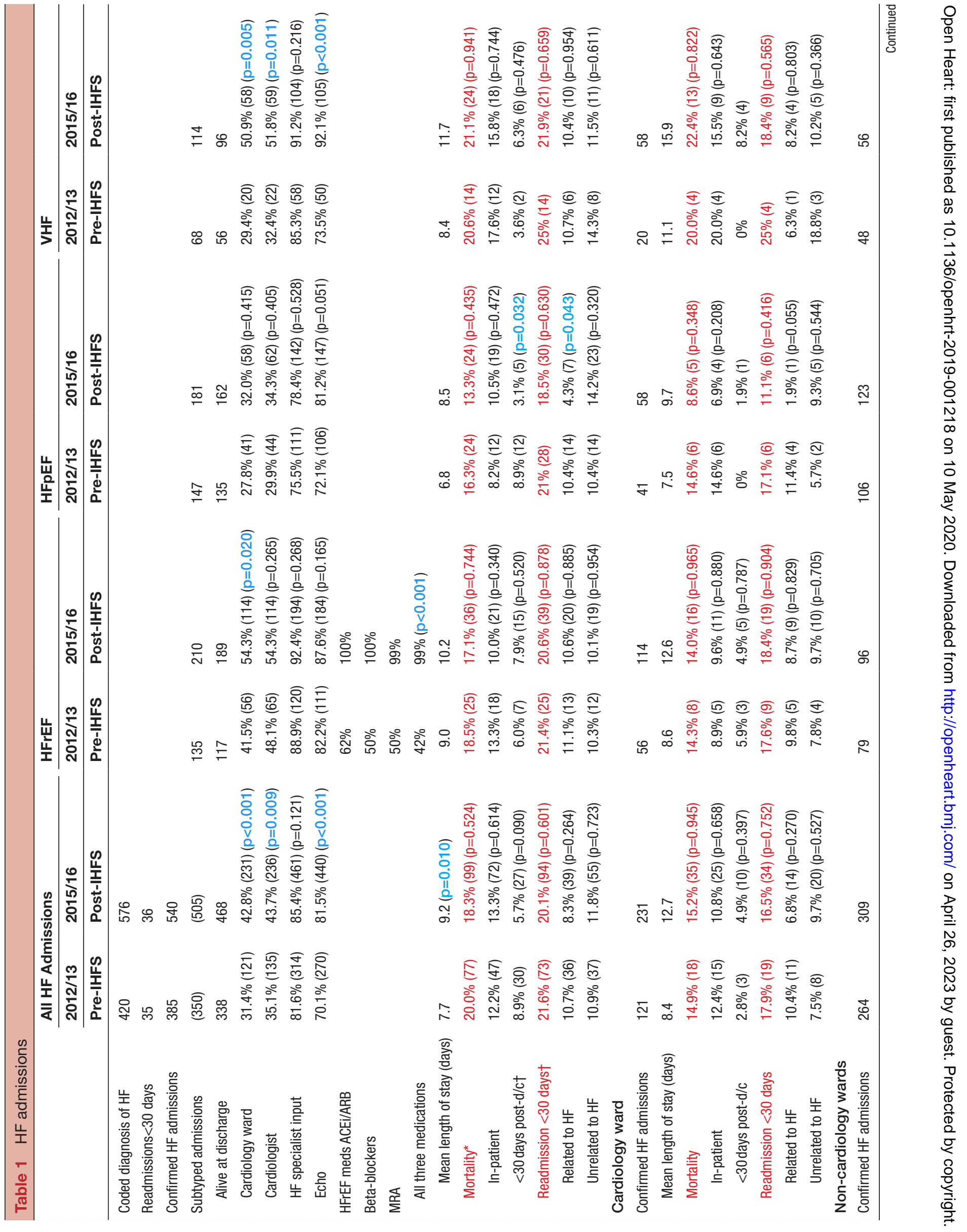




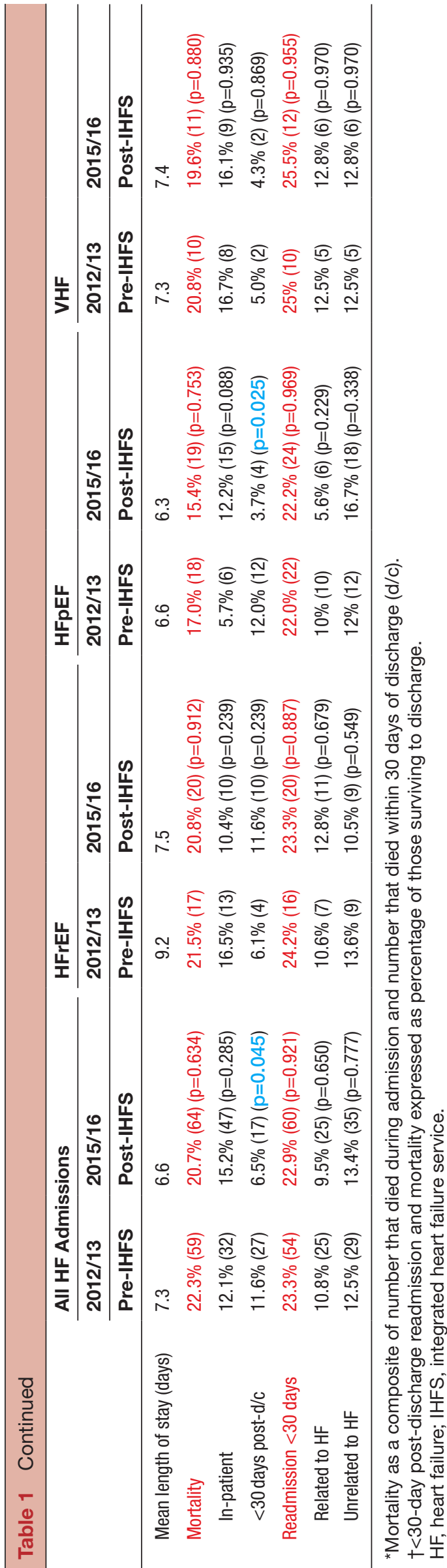

b-blockers and MRA) on discharge significantly increased from 42\% in 2012/2013 to 99\% in 2015/2016 ( $<<0.001$ ). Length of stay in hospital increased from 7.7 to 9.2 days in 2015/2016 ( $\mathrm{p}=0.010)$.

\section{Mortality}

Mortality data is shown in table 1 . The $2015 / 2016$ cohort had a non-significant, lower overall mortality $(20.0 \%$ vs $18.3 \%, \mathrm{p}=0.18$ ), most noticeable in the HFpEF group ( $16.3 \%$ vs $13.3 \%, \mathrm{p}=0.435)$, and to a lesser extent in the HFrEF group ( $18.5 \%$ vs $17.1 \%, \mathrm{p}=0.744)$ but remained high and similar for VHF patients $(20.6 \%$ vs $21.2 \%$, $0.941)$. In-patient mortality was similar in both cohorts. Table 4 shows that the primary cause of death in both cohorts was due to HF (72.3\% in 2012/2013 and 62.5\% in 2015/2016), the remainder often being related to hospital acquired chest infection. Following discharge, 30 -day mortality was reduced by $36 \%$, from $8.9 \%$ to $5.7 \%$ $(\mathrm{p}=0.09)$ in the $2015 / 2016$ cohort, largely driven by a statistically significant $65.2 \%$ reduction in patients with HFpEF (8.9\% vs $3.1 \%$, p=0.032).

\section{Readmissions}

Readmission data is shown in tables 1 and 5. There was a non-significant signal of lower 30-day readmission rate (21.6\% vs $20.1 \%, \mathrm{p}=0.6$ ). This was seen in all three HF subtypes, but was most marked in patients with HFpEF $(21.0 \%$ vs $18.5 \%, \mathrm{p}=0.630)$ and VHF $(25.0 \%$ vs $21.9 \%$, $\mathrm{p}=0.659)$.

The most notable differences were seen in readmissions related to $\mathrm{HF}$ ( $10.7 \%$ vs $8.3 \%, \mathrm{p}=0.264)$, and the reduction reached statistical significance for HF-related readmissions in the HFpEF group ( $10.5 \%$ vs $4.3 \%, \mathrm{p}=0.043)$.

\section{CARDIOLOGY VERSUS NON-CARDIOLOGY Clinical features}

Over the two periods, there was an increase in both the number of patients (121 vs 231) and in the proportion of the total HF admissions managed in the cardiology ward $(31.4 \%$ vs $42.8 \%, \mathrm{p}<0.001)$. Before the introduction of IHFS, patients admitted into the cardiology ward rather than a general medical ward tended to be younger (76 vs 81 years average) and more clinically unwell as judged by proportion of patients in NYHA 3 or 4 (78\% vs $72.7 \%)$, number of co-morbidities, and renal function (mean serum creatinine 133 vs $123 \mathrm{mmol} / \mathrm{L}$ ). By 2015-2016, patients managed in the cardiology ward were of similar ages (78 vs 80 years average), with an even higher proportion in NYHA 3 or $4(91 \%)$, greater co-morbidity, and worse renal function (mean serum creatinine $142 \mathrm{mmol} / \mathrm{L}$ ). Table 2 also shows the change in type of $\mathrm{HF}$ admitted, with a marked increase in numbers of patients with HFrEF and VHF ( HFrEF $+104 \%$, $\mathrm{HFpEF}+41 \%$, VHF $+190 \%$ ). Length of stay was similar in 2012/2013 for patients admitted to the cardiology ward and general medical wards ( 8.4 vs 7.3 days $(\mathrm{p}=0.164)$ but by $2015 / 2016$, had increased dramatically in patients 
Table 2 Morbidity data

\begin{tabular}{|c|c|c|c|c|c|c|c|c|}
\hline & \multicolumn{2}{|c|}{ All HF Admissions } & \multicolumn{2}{|l|}{ HFrEF } & \multicolumn{2}{|l|}{ HFpEF } & \multicolumn{2}{|l|}{ VHF } \\
\hline & $2012 / 2013$ & $2015 / 2016$ & $2012 / 2013$ & $2015 / 2016$ & $2012 / 2013$ & $2015 / 2016$ & $2012 / 2013$ & $2015 / 2016$ \\
\hline & Pre-IHFS & Post-IHFS & Pre-IHFS & Post-IHFS & Pre-IHFS & Post-IHFS & Pre-IHFS & Post-IHFS \\
\hline Coded diagnosis of HF & 420 & 576 & & & & & & \\
\hline Readmissions $<30$ days & 35 & 36 & & & & & & \\
\hline Confirmed HF admissions & 385 & 540 & & & & & & \\
\hline Subtyped admissions & 350 & 505 & 135 & 210 & 147 & 181 & 68 & 114 \\
\hline Age & 81 & 80 & 78 & 77 & 82 & 81 & 83 & 83 \\
\hline Gender F:M & $169: 216$ & $265: 275$ & $49: 85$ & $66: 144$ & $67: 80$ & $110: 71$ & $37: 31$ & $69: 45$ \\
\hline NYHA 1 & $31(8.1 \%)$ & $12(2.2 \%)$ & $7(5 \%)$ & $5(2 \%)$ & $13(9 \%)$ & $3(2 \%)$ & $7(1 \%)$ & $3(3 \%)$ \\
\hline NYHA 2 & $20(5.2 \%)$ & $45(8.3 \%)$ & $10(7 \%)$ & $17(8 \%)$ & $6(4 \%)$ & $11(6 \%)$ & $4(6 \%)$ & $11(10 \%)$ \\
\hline NYHA 3 & $54(14.0 \%)$ & $209(38.7) \%$ & $15(11 \%)$ & $95(45 \%)$ & $21(14 \%)$ & $63(35 \%)$ & $13(19 \%)$ & $44(39 \%)$ \\
\hline NYHA 4 & $233(60.5 \%)$ & $274(50.7 \%)$ & $86(64 \%)$ & $93(44 \%)$ & $89(61 \%)$ & $104(57 \%)$ & $35(51 \%)$ & $56(49 \%)$ \\
\hline Unknown & 47 (12.2\%) & 0 & $17(13 \%)$ & 0 & $18(12 \%)$ & 0 & $9(13 \%)$ & 0 \\
\hline HTN & $140(36.4 \%)$ & $278(51.5 \%)$ & $47(35 \%)$ & $97(46 \%)$ & $48(33 \%)$ & $106(56 \%)$ & $35(51 \%)$ & $56(49 \%)$ \\
\hline Diabetes & $96(24.9 \%)$ & $148(27.4 \%)$ & $41(30 \%)$ & $70(33 \%)$ & $39(27 \%)$ & $56(31 \%)$ & $10(15 \%)$ & $14(13 \%)$ \\
\hline COPD & $58(15 \%)$ & $92(17 \%)$ & $23(17 \%)$ & $36(17 \%)$ & $21(14 \%)$ & $34(19 \%)$ & $9(13 \%)$ & $19(17 \%)$ \\
\hline $\begin{array}{l}\text { Mean serum creatinine } \\
(\mathrm{mmol} / \mathrm{L})\end{array}$ & 123 & 129 & 134 & 145 & 119 & 116 & 116 & 123 \\
\hline HTN unknown & 3 & 0 & 0 & 0 & 3 & 0 & 0 & 0 \\
\hline Diabetes unknown & 2 & 0 & 0 & 0 & 2 & 0 & 0 & 0 \\
\hline COPD unknown & 0 & 0 & 0 & 0 & 0 & 0 & 0 & 0 \\
\hline Admissions to cardiology & 121 & 231 & 56 & 114 & 41 & 58 & 20 & 58 \\
\hline Age & 76 & 78 & 74 & 74 & 79 & 78 & 74 & 83 \\
\hline Gender F:M & $46: 75$ & $103: 128$ & $21: 35$ & $31: 83$ & $17: 24$ & $36: 22$ & $8: 12$ & $36: 22$ \\
\hline NYHA 1 & $13(11 \%)$ & $3(1 \%)$ & $4(7 \%)$ & $1(1 \%)$ & $5(12 \%)$ & $1(2 \%)$ & $3(15 \%)$ & $1(2 \%)$ \\
\hline NYHA 2 & $5(4 \%)$ & $18(8 \%)$ & $4(7 \%)$ & $8(7 \%)$ & 0 & $3(5 \%)$ & $1(5 \%)$ & $7(12 \%)$ \\
\hline NYHA 3 & $17(14 \%)$ & $107(46 \%)$ & $9(16 \%)$ & $56(49 \%)$ & $5(12 \%)$ & $26(45 \%)$ & $3(15 \%)$ & $25(43 \%)$ \\
\hline NYHA 4 & $77(64 \%)$ & $103(45 \%)$ & $36(64 \%)$ & $49(43 \%)$ & $28(68 \%)$ & $28(48 \%)$ & $10(50 \%)$ & $25(43 \%)$ \\
\hline Unknown & $9(7 \%)$ & 0 & $3(5 \%)$ & 0 & $3(7 \%)$ & 0 & $3(15 \%)$ & 0 \\
\hline HTN & $38(31 \%)$ & $113(49 \%)$ & $20(36 \%)$ & $53(46 \%)$ & $8(20 \%)$ & $31(53 \%)$ & $9(45 \%)$ & $28(48 \%)$ \\
\hline Diabetes & $30(25 \%)$ & $63(27 \%)$ & $16(29 \%)$ & $40(35 \%)$ & $10(24 \%)$ & $15(26 \%)$ & $2(10 \%)$ & $8(14 \%)$ \\
\hline COPD & $11(9 \%)$ & $35(15 \%)$ & $6(11 \%)$ & $16(14 \%)$ & $3(7 \%)$ & $10(17 \%)$ & $2(10 \%)$ & $9(16 \%)$ \\
\hline $\begin{array}{l}\text { Mean serum creatinine } \\
(\mathrm{mmol} / \mathrm{L})\end{array}$ & 133 & 142 & 149 & 155 & 117 & 127 & 121 & 132 \\
\hline HTN unknown & 2 & 0 & 0 & 0 & 2 & 0 & 0 & 0 \\
\hline Diabetes unknown & 1 & 0 & 0 & 0 & 1 & 0 & 0 & 0 \\
\hline COPD unknown & 0 & 0 & 0 & 0 & 0 & 0 & 0 & 0 \\
\hline
\end{tabular}

COPD, chronic obstructive pulmonary disease; HF, heart failure; HTN, hypertension; IHFS, integrated heart failure service.

admitted to cardiology vs a general medical ward (12.7 vs 6.6 days, $\mathrm{p}<0.001$ ) (see tables 1 and 2 ).

\section{Mortality}

Table 1 shows lower mortality rates in both cohorts when admitted to a cardiology ward compared with a general medical ward $(33.2 \%$ relative risk reduction in $2012 / 2013,26.6 \%$ in $2015 / 2016)$. This was seen in both HFrEF and HFpEF groups but not in patients with VHF. Of note is the $41 \%$ improvement in 30-day mortality in the $2015 / 2016$ cohort in HFpEF patients managed in the cardiology ward $(14.6 \%$ vs $8.6 \%, \mathrm{p}=0.348)$.

\section{Readmissions}

Readmission rates were lower in patients admitted to cardiology wards in both cohorts. The improvement in readmission rates in $\mathrm{HFpEF}$ patients was driven by the large reduction in $\mathrm{HF}$ related admissions which fell as a proportion from $66.7 \%$ in $2012 / 2013$ to $16.7 \%$ in $2015 / 2016$. 


\begin{tabular}{|c|c|c|c|}
\hline & $2012 / 2013$ & $2015 / 2016$ & \\
\hline & Pre-IHFS & Post-IHFS Y2 & \\
\hline $\begin{array}{l}\text { Post discharge } \\
\text { (7-14 days) }\end{array}$ & $28 \%$ & $57 \%$ & $(p<0.001)$ \\
\hline Routine care & 1104 & 2250 & \\
\hline Home & 489 & 891 & \\
\hline Clinic & 593 & 1371 & \\
\hline Other & 22 & 21 & \\
\hline
\end{tabular}

HFN, heart failure nurse; IHFS, integrated heart failure service; Y2, Second year after IHFS introduction.

\section{DISCUSSION}

HF admissions are increasing and this has been well documented in the UK over the last decade by NICOR. We confirm that over the period 2012/2013-2015/2016 HF hospitalisations at our DGH have increased by $40 \%$. This is likely to reflect not only a real increase in disease prevalence in our ageing population, but also improved patient identification with greater use of BNP and echocardiograms, and better clinical coding practice. The increase was most marked in patients with HFrEF $(55.5 \%)$ and VHF (67.6\%). This may reflect societal and demographic changes, the greater use of echocardiography, and an important coding change with some patients with 'valvular heart disease' now being coded as 'valvular heart failure'.

\section{The new IHFS}

To effectively manage this growing epidemic of increasingly complex HF admissions, we developed and introduced an IHFS at our hospital with the aim of delivering specialist HF care to both hospitalised and community patients. Establishing such a service involved generating a business plan, obtaining financial support for 2.4 WTE additional HFNs, developing an 18-month rotational training programme for our HFNs across hospital and community settings, re-writing consultant job plans, producing local HF management protocols, improving the audit process, establishing an admission alert system, and developing a weekly multi-disciplinary

\begin{tabular}{|c|c|c|}
\hline & $2012 / 2013$ & $2015 / 2016$ \\
\hline & Pre-IHFS & Post-IHFS Y2 \\
\hline IP deaths & 47/385 (13.4\%) & $72 / 540(13.3 \%)$ \\
\hline Heart failure & 34 (72.3\%) & $45(62.5 \%)$ \\
\hline Non-heart failure & $13(27.7 \%)$ & $27(37.5 \%)$ \\
\hline Infection & 11 & 18 \\
\hline ACS & 2 & 7 \\
\hline Cancer & 0 & 2 \\
\hline
\end{tabular}

ACS, acute coronary syndrome; IHFS, integrated heart failure service. meeting, including palliative care involvement. We are pleased to report that the introduction of the IHFS has resulted in more in-patients receiving specialist $\mathrm{HF}$ care delivered by a cardiologist, on a cardiology ward, and with greater use of an early echocardiography. We have also seen a dramatic improvement in the use of the disease-modifying medications for HFrEF (ACEi/ ARB, B-blocker, and MRA) at discharge, increasing from $42 \%$ (of those without contraindications) in 2012/2013 to $99 \%$ in $2015 / 2016(\mathrm{p}<0.001)$. Perhaps as important as this in-patient management is early specialist review post-discharge ${ }^{8}$ and we have significantly increased the number of patients receiving a home visit by an HFN $10-14$ days post-discharge ( $28 \%$ vs $57 \%$, p<0.001).

\section{Outcome data}

A key aim for the IHFS was to assess hard clinical outcome measures in an effort to validate the new model of care. We have shown statistically significant reductions in 30-day mortality and readmission rates in patients with HFpEF. We saw a non-significant reduction in mortality for patients admitted with HF from 2012/2013 (before IHFS) to 2015/2016 (year 3 IHFS).

The principal economic burden of HF relates to the expense of hospitalisation. The introduction of an IHFS has been associated with a non-significant reduction in 30-day readmissions. In 2012/2013 we found readmissions were equally likely to be caused directly by $\mathrm{HF}$ and other non-HF causes. However by $2015 / 2016$, we have demonstrated a $16 \%$ reduction in readmissions due to $\mathrm{HF}$, with most of this benefit seen in patients with HFpEF.

\section{Outcome by HF subtype}

HFrEF

There were improvements in the general aspects of $\mathrm{HF}$ care, with greater specialist input and more patients being managed in the cardiology ward, as well as increased use of disease modifying medications at discharge. We did not observe a statistically significant reduction in mortality or readmission rates. We suspect that this is due to the small sample size, and the fact that prior to the introduction of the IHFS there was already a focus on HFrEF patients, including early follow-up. We believe that the benefit of the higher numbers of patients on disease-modifying medications will be seen beyond 30 days, and will be assessing longer term outcomes in further work.

\section{HFpEF}

Statistically significant reduction in overall 30-day mortality and readmission were seen. These improvements were most pronounced in patients managed on a cardiology ward. This is likely due to better optimisation of fluid status with a greater emphasis on euvolaemia prior to discharge, and related to longer in-patient stays in the cardiology ward.

\section{VHF}

Patients with VHF have poor prognosis and it is unsurprising that mortality in these patients is high and has 
Table 5 Reason for readmission

\begin{tabular}{lcc}
\hline & $\mathbf{2 0 1 2 / 2 0 1 3}$ & $\mathbf{2 0 1 5 / 2 0 1 6}$ \\
\cline { 2 - 3 } Related to HF & Pre-IHFS & Post-IHFS Y2 \\
Decompensation & $36 / 338(10.7 \%)$ & $39 / 468(8.3 \%)$ \\
AKI & $31(86.1 \%)$ & $72.1 \%)$ \\
Unrelated to HF & $5(13.9 \%)$ & $55 / 468(10.9 \%)$ \\
Infection & $37 / 338(10.3 \%)$ & 29 \\
Other cardiac & 13 & $8 \dagger$ \\
Bleeding & $5^{\star}$ & 4 \\
Asthma/COPD (non-infective) & 4 & 1 \\
Falls (no postural drop) & 3 & 2 \\
Other & 2 & $11 \S$ \\
\hline
\end{tabular}

${ }^{*}$ Three due to atrial fibrillation, one non-ST-segment elevation myocardial infarction (NSTEMI), one angina.

†Three due to angina, two atrial flutter, one atrioventricular block, onesupraventricular tachycardia, one NSTEMI.

$\ddagger$ Acute ischaemia, acute kidney injury (AKI) (not taking nephrotoxics), constipation, chronic ischaemia, diverticulosis, lung cancer,

musculoskeletal pain, no clear pathology, retention, spinal stenosis.

§Bowel obstruction, confusion, constipation, delirium, erratic blood glucose, gout, hypoglycaemia, no clear pathology, non-cardiac chest pain, reduced conscious level, submandibular gland swelling.

HF, heart failure; IHFS, integrated heart failure service.

remained unchanged across our two cohorts. There was a non-significant reduction in readmissions, but due to inconsistent coding of these patients in 2012/2013 it is difficult to draw any conclusions from this. We plan to look in more detail at this group, and whether more of them should be considered for early surgical intervention.

\section{Cardiology versus non-cardiology ward care}

Our data point to the benefit of early, specialist cardiology input and admission to a cardiology ward on 30-day mortality and readmissions at the expense of longer in-patient stay.

For many years, cardiologists and HF specialists have focused much of their efforts on HFrEF as it is this subtype for which we are currently able to provide effective pharmacological and device-based therapies. Our study showed the greatest impact on patients with HFpEF, both with reduced early mortality, and HF readmissions. Benefits were greatest in patients managed on a cardiology ward with specialist HF input.

We suggest that this may be related to more aggressive and optimised in-patient diuresis, better management of atrial fibrillation and other risk factors, improved training in self-management, and better provision of early postdischarge review and continuing care by a specialist HFN. We intend to study this in greater detail.

For patients with VHF we believe that outcomes can be improved with early identification and discussion with the cardiothoracic team. Work is on-going to assess the impact that the IHFS has on identifying and treating this group of patients.

\section{Limitations}

1. As an observational single centre study with relatively small patient numbers, our study was underpowered to show statistically significant differences in the major outcomes.

2. We were unable to confirm the coding diagnosis of $\mathrm{HF}$ in some patients from each cohort although this represents only a small proportion of the patients assessed.

3. Some patients did not have an echocardiogram and therefore could not be subtyped.

4. BNP was not routinely used in all patients but is used in a targeted fashion in patients with clinical concerns or diagnostic uncertainty.

\section{CONCLUSION}

Since the introduction of our IHFS we have been pleased to see improved identification of patients admitted with $\mathrm{HF}$, higher levels of specialist $\mathrm{HF}$ in-patient care, improved use of disease-modifying HFrEF medications, and a greater number of patients benefiting from early HFN follow-up. Despite increasing admission numbers, of sicker patients, we have seen improved 30-day mortality and HF-related readmission rates in the HFpEF cohort. This is particularly encouraging, given how difficult it is to improve outcomes for this group. An IHFS can be developed and introduced without major resources and with simple service re-design and could be applicable across the NHS to improve HF care and clinical outcomes.

Acknowledgements James Edgar contributed to data collection. Bev Coomesas lead heart failure nurse in the Hospital—offered significant advice and information regarding the service.

Contributors $\mathrm{AH}$ was the main contributor to the acquisition and analysis of the study data, as well as taking a leading role in the final write-up. JH collected and analysed data and was responsible for large sections of the write-up, as well as critically assessing and revising the write-up. AC contributed significantly to the data collection and was involved in the planning and analysis. SI contributed 
significantly to the data collection and analysis as well as writing-up sections of the final paper. PK has instigated the new IHFS and supervised this project. He contributed to the data collection, and contributed significantly to the analysis, results and discussion sections.

Funding The authors have not declared a specific grant for this research from any funding agency in the public, commercial or not-for-profit sectors.

Competing interests None declared.

Patient consent for publication Not required.

Ethics approval Ethical approval was not required for this project, since the data were anonymised patient data that has already been collected and submitted to the NICOR heart failure audit.

Provenance and peer review Not commissioned; externally peer reviewed.

Data availability statement Data are available upon reasonable request. All data relevant to the study are included in the article or uploaded as supplementary information. We used anonymised patient data, that had been collated into an excel spreadsheet by the audit team and submitted to NICOR as part of the national heart failure audit. Anonymised data for both study cohorts is available from request from the corresponding author (orcid id 0000-0003-0422-9147).

Open access This is an open access article distributed in accordance with the Creative Commons Attribution Non Commercial (CC BY-NC 4.0) license, which permits others to distribute, remix, adapt, build upon this work non-commercially, and license their derivative works on different terms, provided the original work is properly cited, appropriate credit is given, any changes made indicated, and the use is non-commercial. See: http://creativecommons.org/licenses/by-nc/4.0/.

ORCID iD

Alasdair Hawley http://orcid.org/0000-0003-0422-9147

\section{REFERENCES}

1 Cowie MR, Wood DA, Coats AJ, et al. Survival of patients with a new diagnosis of heart failure: a population based study. Heart 2000;83:505-10

2 Hobbs FDR, Roalfe AK, Davis RC, et al. Prognosis of all-cause heart failure and borderline left ventricular systolic dysfunction: 5 year mortality follow-up of the echocardiographic heart of England screening study (ECHOES). Eur Heart J 2007;28:1128-34.

3 Azad N, Lemay G. Management of chronic heart failure in the older population. J Geriatr Cardiol 2014;11:329-37.

4 British Society for Heart Failure. National heart failure audit (April 2014-March 2015). Available: https://www.ucl.ac.uk/nicor/audits/ heartfailure/documents/annualreports/heartfailurepublication14_15 [Accessed 28 Nov 2016].

5 Torbay Hospital CQC report. Available: https://www.cqc.org.uk/ location/RA901/reportsort

6 Pathirana $\mathrm{TI}$, Jackson CA. Socioeconomic status and multimorbidity: a systematic review and meta-analysis. Aust N Z J Public Health 2018;42:186-94.

7 National Institute for Health and Care Excellence (NICE). Management of chronic heart failure in adults in primary and secondary care, 2010. Available: https://wwwniceorguk/guidance/cg108/resources/ guidance-chronic-heart-failure-pdf

8 Gandhi S, Mosleh W, Sharma UC, et al. Multidisciplinary heart failure clinics are associated with lower heart failure hospitalization and mortality: systematic review and meta-analysis. Can J Cardiol 2017:33:1237-44

9 Ponikowski P, Voors AA, Anker SD, et al. 2016 ESC Guidelines for the diagnosis and treatment of acute and chronic heart failure: The Task Force for the diagnosis and treatment of acute and chronic heart failure of the European Society of Cardiology (ESC)Developed with the special contribution of the Heart Failure Association (HFA) of the ESC. Eur Heart J 2016;37:2129-200. 